\title{
CLULEX at SemEval-2021 Task 1: A Simple System Goes a Long Way
}

\author{
Greta Smolenska, Peter Kolb, Sinan Tang, \\ Mironas Bitinis, Héctor Hernández, Elin Asklöv \\ Babbel|Lesson Nine GmbH \\ \{gsmolenska, pkolb, stang, mbitinis, hhernandez, easkloev\}@babbel.com
}

\begin{abstract}
This paper presents the system we submitted to the first Lexical Complexity Prediction (LCP) Shared Task 2021. The Shared Task provides participants with a new English dataset that includes context of the target word. We participate in the single-word complexity prediction sub-task and focus on feature engineering. Our best system is trained on linguistic features and word embeddings (Pearson's score of 0.7942). We demonstrate, however, that a simpler feature set achieves comparable results and submit a model trained on 36 linguistic features (Pearson's score of 0.7925).
\end{abstract}

\section{Introduction}

Lexical complexity relates to complexity of words. Its assessment can be beneficial in a number of fields, ranging from education to communication. For instance, lexical complexity studies can assist in providing language learners with learning materials suitable for their proficiency level or aid in text simplification (Siddharthan, 2014). These studies are also a central part of reading comprehension, as lexical complexity can predict which words might be difficult to understand and could hinder the readability of the text. Lexical complexity studies typically make use of Natural Language Processing and Machine Learning methods (Paetzold and Specia, 2016).

Previous similar studies focus on Complex Word Identification (CWI), which is a process of identifying complex words in a text (Shardlow, 2013). In this case, lexical complexity is assumed to be binary - words are either complex or not. LCP Shared Task 2021 addresses this limitation by introducing a new dataset designed for continuous rather than binary complexity prediction (Shardlow et al., 2021).

In this paper, we describe a single-word lexical complexity prediction system. Our goal is to demonstrate that a simple system can achieve results comparable to more complex ones. Therefore, we focus on feature engineering rather than model tuning.

\section{Related Work}

\subsection{Lexical Complexity}

Over the years, studies on lexical complexity have ranged from research on the overall readability enhancement and text simplification to studies focusing specifically on lexical complexity.

Some of the earlier work on lexical complexity targeted communication enhancement of medical documents by assessing the familiarity of medical terminology (Zeng et al., 2005). Paetzold and Specia (2013) showed that the absence of lexical simplification in Automatic Text Simplification (ATS) systems yielded texts that readers might still find too complex to understand.

CWI has then gained more interest, and two Shared Tasks have been organised with the goal of establishing state-of-the-art performance in the field. SemEval-2016 Task 11 approached CWI as a binary classification task and collected a dataset for English which was annotated by non-native speakers (Paetzold and Specia, 2016). Zampieri et al. (2017) showed that such data annotation approach was not optimal. The second Shared Task addressed the limitations by introducing a multilingual dataset for Spanish, German, English and French and approaching the problem as both, a $b i$ nary and a probabilistic complexity prediction task (Štajner et al., 2018).

\subsection{Feature and Model Selection}

In lexical complexity prediction tasks, linguistic features and word frequency measures have been proven to be among the most effective features. The winning systems developed for the CWI 2018 
Shared Task (Yimam et al., 2018) use various lexical features, such as word N-gram, POS tags, and syntactic dependency parse relations. Moreover, they also include different variants of word frequency features, CEFR levels, and a few more.

As for the choice of algorithms, Gooding and Kochmar (2018) has achieved the best performing systems in English monolingual tasks using classifiers with ensemble techniques, such as AdaBoost with 5000 estimators and the aggregation classifier of Random Forest. The winning systems for multilingual tracks (Kajiwara and Komachi, 2018) also employ random forest models.

\section{LCP Shared Task 2021 Setup}

The LCP Shared Task 2021 aims to predict the complexity value of words in their context. It is divided into two sub-tasks: predicting the complexity score of 1) single words and 2) multi-word expressions. In this paper, we present a system for the first sub-task.

The Shared Task uses the CompLex corpus (Shardlow et al., 2020). In addition to the target word, it includes contextual information which is represented by a sentence where the word appears and its source or domain: Bible (Christodouloupoulos and Steedman, 2015), Europarl (Koehn, 2005) or biomedical texts (Bada et al., 2012). Each word in the dataset is evaluated by around 7 annotators from English speaking countries. The complexity labels are based on a 5-point Likert scale scheme (very easy to very difficult). The final dataset consists of 7,662 training and 917 testing instances.

The Shared Task baseline system uses a linear regression model. It is trained on $\log$ relative frequency and word length features, resulting in a Mean Absolute Error (MAE) of $\mathbf{0 . 0 8 6 7}$.

\section{Methodology}

In this section, we describe the methodology that we follow in the design of our system, including the used data, feature engineering and the training steps. The study relies on an in-depth experimentation with features. We aim to find out which linguistic information is the best predictor of lexical complexity.

\subsection{Data Collection}

For the computation of some features, we use additional data sources. We extract word frequencies from nine corpora that cover different do- mains and complexity levels: $B N C$ corpus $^{1}$, Simple Wikipedia and English Wikipedia ${ }^{2}$, SubIMDB $^{3}$ and English monolingual corpora from the OPUS project $^{4}$ : bible-uedin $^{5}$, EMEA $^{6}$, Europarl ${ }^{7}$, NewsCommentary $^{8}$ and OpenSubtitles $2018^{9}$. We additionally use two word lists with annotated CEFR levels (Common European Framework of Reference for Languages, which organises language proficiency in six levels, A1 to C2 $)^{10}$ and the Age of Acquisition dataset ${ }^{11}$.

\subsection{Features}

We consider a) word and sentence-level features (or linguistic features), b) frequency features and c) word embeddings.

On a word level, we compute the linguistic information, i.e. character, syllable and phoneme counts, universal part-of-speech tag and named entity tag (extracted with Stanza NLP toolkit) (Qi et al., 2020). We also compute scores that pertain to language learning such as age of acquisition, percentage of population that knows the word and word prevalence (Kuperman et al., 2012). Finally, we use two CEFR word lists and split them into five subsets each (one per CEFR level). Each word is assigned a boolean value depending whether it appears in one of the subsets.

On a sentence level, lexical complexity is represented by lexical diversity rate (unique words divided by all words). Syntactic complexity and readability are represented by the average sentence length and the Linsear Write score, which is a readability measure used to assess the difficulty of U.S. military manuals (Klare, 1974). We also make special use of the OpenSubtitles frequencies: vocabulary percentage per CEFR level is computed by splitting the corpus into five subsets and represents the distribution of words among the five frequency ranges; difficult word percentage relates to words containing two and more syllables that do not appear in top 200 most common words in the corpus; unknown word percentage represents

\footnotetext{
${ }^{1} \mathrm{BNC}$

${ }^{2}$ Wikipedia Monolingual Corpora

${ }^{3}$ SubIMDB

${ }^{4}$ OPUS resources

${ }^{5}$ bible-uedin

${ }^{6}$ EMEA

${ }^{7}$ Europarl

${ }^{8}$ News-Commentary

${ }^{9}$ OpenSubtitles 2018

${ }^{10}$ The Oxford 5000 and Kelly list for English

${ }^{11} \mathrm{AoA}$
} 
the percentage of words that do not appear in the corpus at all. The final text complexity score is a normalised sum of all sentence-level scores.

Additionally, we calculate different types of frequencies, i.e. log relative, absolute (raw), frequency rank (word rank in a frequency list) and ZIPF frequency (Zipf, 1949), from the nine corpora.

Finally, we experiment with pre-trained word embeddings, including fastText for English and BERT's embeddings (Mikolov et al., 2018; Devlin et al., 2018). However, we ablate fastText word embeddings from the final feature set as they slightly degrade the overall performance.

\subsection{Training, Tuning \& Testing}

The focus of our study is to achieve the best results through feature engineering rather than model hyperparameter tuning. During all experiments, we utilise the open source Machine Learning software WEKA (Frank et al., 2016) with the default algorithm hyperparameter settings and apply 10-fold cross-validation.

\subsubsection{Models}

First, we select several Machine Learning algorithms for further experiments with the features. During this step, we use word and sentence-level features with a subset of frequency features.

Due to the nature of the dataset target values, we employ classifiers suitable for regression tasks. Specifically, we use linear regression and Multi-Layer Perceptron, meta classifiers, such as Bagging, Stacking and Random Subspace, and decision trees, such as M5P and Random Forest. We obtain the best result and benchmark our approach with M5P - a model tree algorithm used for numeric prediction (Table 1). We reach MAE of $\mathbf{0 . 0 6 3 8}$ (Pearson's score of 0.7811 ), outperforming the baseline model of the Shared Task (Section 3).

Next, we experiment with different feature groups and combinations with the goal to select the optimal feature subset. We train with the five best performing algorithms in each step but report only the results of the best model.

\subsubsection{Ablation Studies}

We narrow down the selection for the best performing features based on the three feature groups: frequency features, linguistic features and word embeddings.

\begin{tabular}{l|l|l}
\hline \hline Classifier & Pearson & MAE \\
\hline M5P & $\mathbf{0 . 7 8 1 1}$ & $\mathbf{0 . 0 6 3 8}$ \\
Random SubSpace & 0.77 & 0.0657 \\
Bagging & 0.7693 & 0.0657 \\
Random Forest & 0.7655 & 0.0661 \\
Decision Table & 0.7601 & 0.0665 \\
\hline \hline
\end{tabular}

Table 1: 10-fold cross-validation results on the training set for the top 5 classifiers

We pay special attention to frequency features since the previous work shows that word frequencies are usually among the most informative features (Yimam et al., 2018). First, to figure out the best way to represent frequencies of lower cased word forms, we train the M5P model on different frequency representations: $\log$ relative, raw, ZIPF and frequency rank. We use only the best frequency representation, log relative frequency, in the following steps. We then test the models with frequencies from various sources.

We also conduct experiments to understand the impact of word embeddings using 300-dimension pre-trained word vectors ${ }^{12}$, and BERT $^{13}$ embeddings, where we concatenate layers 7 and 11 (Chronis and Erk, 2020) which gives better results than concatenating or summing the last four hidden layers.

We then conduct the final ablation study. Given the complete set of features, we employ WEKA's feature selection algorithms and remove the least informative features, one feature at a time. In case it does not result in an improvement, the feature is added back and we continue with the next available feature.

\section{Results}

In this section, we present our results and discuss the key findings. All discussed systems are trained with the Random Forest classifier.

\subsection{Frequency Features}

We find that a combination of frequency features from different sources alone can result in high performance (Table 2). In this case, daily spoken language sources, such as film subtitles, seem to be the most informative. However, adding more frequency features does not necessarily improve the results (Tables 2 and 3 ).

\footnotetext{
${ }^{12}$ fastText for English

${ }^{13}$ We use bert-base-uncased from Hugging Face (Wolf et al., 2020)
} 


\begin{tabular}{l|l}
\hline \hline Frequency Sources & Pearson \\
\hline All - EMEA & $\mathbf{0 . 7 1 3}$ \\
All & 0.7128 \\
All - EMEA - Bible & 0.7041 \\
OpenSubs + BNC & 0.6882 \\
+ EnWiki + SimpleWiki & 0.6536 \\
OpenSubs & 0.6479 \\
SubIMDB & \\
\hline \hline
\end{tabular}

Table 2: Frequency Sources

\begin{tabular}{l|l}
\hline \hline Features & Pearson \\
\hline $\begin{array}{l}\text { 9 frequencies + corpus + POS + } \\
\text { syllCount + charCount (13 features) }\end{array}$ & 0.764 \\
Above + BERT 7-11 (1550 features) & 0.6953 \\
9 frequencies + corpus + POS & \\
+ sentence features - depRel - distToHead & 0.7907 \\
- NER (44 features) & \\
Above - imdbFreq & 0.7909 \\
Above - CEFR vocabulary percentages & 0.7921 \\
Above - freqPm & 0.7924 \\
Above - harmonicMeanDiff (36 features) & $\mathbf{0 . 7 9 2 5}$ \\
Above + best BERT 7-11 (76 features) & $\mathbf{0 . 7 9 4 2}$ \\
\hline \hline
\end{tabular}

Table 3: Feature Ablation Experiments

\subsection{Linguistic Features}

During the experiments with the linguistic features, we obtain the best results using a reduced 36 feature combination (Table 4). We find out that syntactic features such as target word distance to the syntactic head of the sentence and its syntactic relation to the head of the sentence seem to worsen the performance (Table 3). The full list of ablation steps can be found in Appendix A.

Furthermore, removing the sentence-level features results in a slight decrease of the overall performance (from Pearson's score of 0.7925 to $0.7791)$. It indicates that either word-level information remains the most informative for this task or that a single sentence does not provide sufficient contextual information.

\subsection{Word Embedding Features}

Table 4 shows results for the best systems that are trained on linguistic features only, word embedding features only and the combined set of features.

The system trained on the word embeddings performs significantly worse than the other two systems. BERT embeddings only improve the result if we select a subset of 76 out of the 1536 embedding features with WEKA's CfsSubsetEval (Hall, 1999). The model trained on the combined set of features performs the best, reaching Pearson's score of $\mathbf{0 . 7 9 4 2}$. However, the difference between this system and the one trained on linguistic fea-

\begin{tabular}{l|l|l}
\hline \hline Feature Combination & \#Features & Pearson \\
\hline 36 Linguistic + & 112 & $\mathbf{0 . 7 9 4 2}$ \\
76 Embedding & 36 & 0.7925 \\
Linguistic & 1536 & 0.6999 \\
BERT Embeddings & \\
\hline
\end{tabular}

Table 4: Best systems trained on linguistic, word embedding and the combined features

tures is statistically insignificant. These results indicate that word embeddings are less informative than the linguistic information. Additionally, word embedding computation can be costly in terms of the added complexity and the computational resources. We, therefore, argue that a simpler feature combination is sufficient and submit our second best model to the Shared Task.

\subsection{Test Set}

The submitted system that is trained on 36 linguistic features (Appendix B) is evaluated on the official Shared Task test set and reaches Pearson's score of $\mathbf{0 . 7 5 8 8}$, ranking in the upper half of the submitted systems.

\section{Conclusion}

In this paper, we have described the design of our system submitted to the LCP Shared Task 2021 and discussed the key findings of our feature engineering approach. We aimed to design a simple system that would not require much classifier tuning or complex feature computations. Our two best models are trained on the Random Forest classifier with the default hyperparameters. The best system is trained on a 112 feature set which includes word embeddings. The second best system is trained on a simple 36 linguistic feature set. We submit the simple system since the performance difference between the two systems is not significant. The model is placed in the upper half of the Shared Task rankings for the single-word prediction subtask (Pearson's score of 0.7588), demonstrating how a simple approach can achieve high performance results.

Further analysis of the feature ablation studies confirms that word frequencies seem to be the most informative among all features. We also observe that even though including contextual information does improve the overall result, the performance differences are small. Future research might therefore look into including more contextual information than one sentence. In addition, the perception 
of word complexity differs from reader to reader. Future work could target specific reader groups, such as people with dyslexia or second language learners. In this case, the relevant background information of the readers should be included in the annotation and experimentation processes.

\section{References}

Michael Bada, Miriam Eckert, Donald Evans, Kristin Garcia, Krista Shipley, Dmitry Sitnikov, William A Baumgartner, K Bretonnel Cohen, Karin Verspoor, Judith A Blake, et al. 2012. Concept annotation in the CRAFT corpus. BMC bioinformatics, 13(1):120.

Christos Christodouloupoulos and Mark Steedman. 2015. A massively parallel corpus: The Bible in 100 languages. Language resources and evaluation, 49(2):375-395.

Gabriella Chronis and Katrin Erk. 2020. When is a bishop not like a rook? When it's like a rabbi! Multiprototype BERT embeddings for estimating semantic relationships. In Proceedings of the 24th Conference on Computational Natural Language Learning, pages 227-244.

Jacob Devlin, Ming-Wei Chang, Kenton Lee, and Kristina Toutanova. 2018. BERT: Pre-training of deep bidirectional transformers for language understanding. arXiv preprint arXiv:1810.04805.

Eibe Frank, Mark A. Hall, and Ian H. Witten. 2016. The WEKA Workbench. Morgan Kaufmann. Fourth Edition.

Sian Gooding and Ekaterina Kochmar. 2018. CAMB at CWI shared task 2018: Complex word identification with ensemble-based voting. In Proceedings of the Thirteenth Workshop on Innovative Use of NLP for Building Educational Applications, pages 184-194, New Orleans, Louisiana. Association for Computational Linguistics.

Mark Andrew Hall. 1999. Correlation-based feature selection for machine learning.

Tomoyuki Kajiwara and Mamoru Komachi. 2018. Complex word identification based on frequency in a learner corpus. In Proceedings of the Thirteenth Workshop on Innovative Use of NLP for Building Educational Applications, pages 195-199, New Orleans, Louisiana. Association for Computational Linguistics.

George R Klare. 1974. Assessing readability. Reading research quarterly, pages 62-102.

Philipp Koehn. 2005. Europarl: A parallel corpus for statistical machine translation. In MT summit, volume 5, pages 79-86. Citeseer.
Victor Kuperman, Hans Stadthagen-Gonzalez, and Marc Brysbaert. 2012. Age-of-acquisition ratings for 30,000 English words. Behavior research methods, 44(4):978-990.

Tomas Mikolov, Edouard Grave, Piotr Bojanowski, Christian Puhrsch, and Armand Joulin. 2018. Advances in pre-training distributed word representations. In Proceedings of the International Conference on Language Resources and Evaluation (LREC 2018).

Gustavo Paetzold and Lucia Specia. 2013. Text simplification as tree transduction. In Proceedings of the 9 th Brazilian Symposium in Information and Human Language Technology.

Gustavo Paetzold and Lucia Specia. 2016. SemEval 2016 task 11: Complex word identification. In Proceedings of the 10th International Workshop on Semantic Evaluation (SemEval-2016).

Peng Qi, Yuhao Zhang, Yuhui Zhang, Jason Bolton, and Christopher D. Manning. 2020. Stanza: A Python Natural Language Processing Toolkit for many human languages. In Proceedings of the 58th Annual Meeting of the Association for Computational Linguistics: System Demonstrations.

Matthew Shardlow. 2013. A comparison of techniques to automatically identify complex words. In 51st Annual Meeting of the Association for Computational Linguistics Proceedings of the Student Research Workshop, pages 103-109, Sofia, Bulgaria. Association for Computational Linguistics.

Matthew Shardlow, Michael Cooper, and Marcos Zampieri. 2020. CompLex-A new corpus for lexical complexity predicition from Likert scale data. arXiv preprint arXiv:2003.07008.

Matthew Shardlow, Richard Evans, Gustavo Paetzold, and Marcos Zampieri. 2021. SemEval-2021 task 1: Lexical complexity prediction. In Proceedings of the 14th International Workshop on Semantic Evaluation (SemEval-2021).

Advaith Siddharthan. 2014. A survey of research on text simplification. ITL-International Journal of Applied Linguistics, 165(2):259-298.

Sanja Štajner, Chris Biemann, Shervin Malmasi, Gustavo Paetzold, Lucia Specia, Anaïs Tack, Seid Muhie Yimam, and Marcos Zampieri. 2018. A report on the complex word identification shared task 2018. In Proceedings of the 13th Workshop on Innovative Use of NLP for Building Educational Applications.

Thomas Wolf, Julien Chaumond, Lysandre Debut, Victor Sanh, Clement Delangue, Anthony Moi, Pierric Cistac, Morgan Funtowicz, Joe Davison, Sam Shleifer, et al. 2020. Transformers: State-of-theart natural language processing. In Proceedings of the 2020 Conference on Empirical Methods in Natural Language Processing: System Demonstrations, pages 38-45. 
Seid Muhie Yimam, Chris Biemann, Shervin Malmasi, Gustavo Paetzold, Lucia Specia, Sanja Štajner, Anaïs Tack, and Marcos Zampieri. 2018. A report on the complex word identification shared task 2018. In Proceedings of the Thirteenth Workshop on Innovative Use of NLP for Building Educational Applications, pages 66-78, New Orleans, Louisiana. Association for Computational Linguistics.

Marcos Zampieri, Shervin Malmasi, Gustavo Paetzold, and Lucia Specia. 2017. Complex word identification: Challenges in data annotation and system performance. In Proceedings of the 4th Workshop on Natural Language Processing Techniques for Educational Applications (NLPTEA 2017).

Qing Zeng, Eunjung Kim, Jon Crowell, and Tony Tse. 2005. A text corpora-based estimation of the familiarity of health terminology. In International Symposium on Biological and Medical Data Analysis, pages 184-192. Springer.

George K. Zipf. 1949. Human Behaviour and the Principle of Least Effort. Addison-Wesley. 


\section{Appendices}

\section{A Feature Ablation Experiments}

\begin{tabular}{|c|c|c|c|}
\hline Features & \#Features & Pearson & $\begin{array}{l}\text { Features } \\
\text { perma- } \\
\text { nently } \\
\text { removed }\end{array}$ \\
\hline $\begin{array}{l}9 \text { frequencies + corpus } \\
+ \text { POS }+ \text { syllCount }+ \\
\text { charCount }\end{array}$ & 13 & 0.764 & \\
\hline Above + BERT 7-11 & 1550 & 0.6953 & \\
\hline $\begin{array}{l}9 \text { frequencies + cor- } \\
\text { pus + POS + sentence- } \\
\text { level features (- deprel } \\
\text { - distToHead - NER) }\end{array}$ & 44 & 0.7907 & yes \\
\hline $\begin{array}{l}\text { above }+300 \text { fastText } \\
\text { word embeddings }\end{array}$ & 344 & 0.766 & \\
\hline 44 - imdbFreq & 43 & 0.7909 & yes \\
\hline $\begin{array}{l}43 \text { - Oxford lists - } \\
\text { Kelly lists }\end{array}$ & 32 & 0.7902 & \\
\hline $43-\mathrm{AoA}$ & 42 & 0.7891 & \\
\hline $\begin{array}{l}43 \text { - CEFR vocabulary } \\
\text { percentages }\end{array}$ & 38 & 0.7921 & yes \\
\hline $\begin{array}{l}38-\text { avgSentence- } \\
\text { Length }\end{array}$ & 37 & 0.792 & \\
\hline 38 - linsearWrite & 37 & 0.7917 & \\
\hline $\begin{array}{l}38 \text { - unknownWord- } \\
\text { Percentage }\end{array}$ & 37 & 0.7906 & \\
\hline $\begin{array}{l}38 \text { - difficultWordPer- } \\
\text { centage }\end{array}$ & 37 & 0.7914 & \\
\hline $\begin{array}{l}38-\text { lexicalDiversi- } \\
\text { tyRate }\end{array}$ & 37 & 0.792 & \\
\hline $\begin{array}{l}38-\text { textComplexi- } \\
\text { tyScore }\end{array}$ & 37 & 0.7919 & \\
\hline 38 - countPhones & 37 & 0.7918 & \\
\hline 38 - percKnown & 37 & 0.7908 & \\
\hline 38 - freqPm & 37 & 0.7924 & yes \\
\hline 37 - prevalence & 36 & 0.79 & \\
\hline 37 - freqZipfUS & 36 & 0.7923 & \\
\hline 37 - avgDiffRating & 36 & 0.7923 & \\
\hline $\begin{array}{l}37 \text { - harmonicMean- } \\
\text { DiffRating }\end{array}$ & 36 & 0.7925 & yes \\
\hline $\begin{array}{l}36+\text { best BERT 7-11 } \\
\text { (76) }\end{array}$ & 112 & 0.7942 & yes \\
\hline
\end{tabular}




\section{B Final Feature Set}

\begin{tabular}{|c|c|}
\hline Feature & Description \\
\hline corpus & $\begin{array}{l}\text { One of }\{\text { bible, biomed, eu- } \\
\text { roparl }\}\end{array}$ \\
\hline POS & Part-of-speech tag \\
\hline linsearWrite & $\begin{array}{l}\text { readability measure used in U.S. } \\
\text { military }\end{array}$ \\
\hline avgSentenceLength & number of words in the sentence \\
\hline unknownWordPercentage & unknown word percentage \\
\hline difficultWordPercentage & difficult word percentage \\
\hline lexicalDiversityRate & $\begin{array}{l}\text { type token ratio (unique } \\
\text { words/all words) }\end{array}$ \\
\hline textComplexityScore & $\begin{array}{l}\text { normalised sum of all sentence- } \\
\text { level scores }\end{array}$ \\
\hline countPhones & count of phones in word \\
\hline AoA & age of acquisition \\
\hline percKnown & $\begin{array}{l}\text { Percentage of population that } \\
\text { knows the word. }\end{array}$ \\
\hline prevalence & word prevalence \\
\hline freqZipfUS & $\begin{array}{l}\text { ZIPF frequency calculated from } \\
\text { the AoA dataset }\end{array}$ \\
\hline avgDiffRating & $\begin{array}{l}\text { Average of difficulty ratings } \\
\text { from SVL } 12000 \text { dataset }\end{array}$ \\
\hline $\begin{array}{l}\text { kelly_a1 } \\
\text { oxford_a1 } \\
\text { kelly_a2 } \\
\text { oxford_a2 } \\
\text { kelly_b1 } \\
\text { oxford_b1 } \\
\text { kelly_b2 } \\
\text { oxford_b2 } \\
\text { kelly_c1 } \\
\text { oxford_c1 } \\
\text { kelly_c2 }\end{array}$ & $\begin{array}{l}\text { boolean: } \\
\text { for } \\
\text { word } \\
\text { that } \\
\text { occurs } \\
\text { in } \\
\text { the } \\
\text { CEFR } \\
\text { wordlist }\end{array}$ \\
\hline syllCount & number of syllables in the word \\
\hline charCount & $\begin{array}{l}\text { number of characters in the } \\
\text { word }\end{array}$ \\
\hline $\begin{array}{l}\text { Europarl_log_rel_freq } \\
\text { BNC_log_rel_freq } \\
\text { OpenSubs_log_rel_freq } \\
\text { SimpleWiki_log_rel_freq } \\
\text { EnWiki_log_rel_freq } \\
\text { SubIMDB_log_rel_freq } \\
\text { News_Comm_log_rel_freq } \\
\text { bible_log_rel_freq }\end{array}$ & $\begin{array}{l}\text { log } \\
\text { relative } \\
\text { frequency } \\
\text { of } \\
\text { word } \\
\text { in } \\
\text { the } \\
\text { corpus }\end{array}$ \\
\hline complexityTargetClass & numeric \\
\hline
\end{tabular}

